\title{
Revision of phylogenic relationships between several Acrididae subfamilies
}

\author{
Sukhikh I. ${ }^{1 *}$, Ustyantsev K. ${ }^{1}$, Bugrov A. ${ }^{2,3}$, Sergeev M. ${ }^{2,3}$, Vavilova V. ${ }^{1}$, Blinov A. ${ }^{1}$ \\ ${ }^{1}$ Institute of Cytology and Genetics, SB RAS, Novosibirsk, Russia \\ ${ }^{2}$ Novosibirsk State University, Novosibirsk, Russia \\ ${ }^{3}$ Institute of Systematics and Ecology of Animals, SB RAS, Novosibirsk, Russia \\ *e-mail: igor3419@gmail.com
}

Key words: Orthoptera, Acrididae, molecular phylogeny, insects

Motivation and Aim: Acrididae, with more than 6,500 species, is the largest, cosmopolitan family of short-horned orthopteran insects (Orthoptera, Caelifera). Due to their importance as agricultural pests, Acrididae had long attracted the attention of many taxonomists. However, there are many disagreements about position and status of several subfamilies of Acrididae family. Analysing sequences of several phylogenetic markers from the NCBI GenBank database, with the addition of experimentally obtained data, we try to determine the phylogenetic relation and taxonomic rank of the problematic groups.

Methods and Algorithms: Sequences of complete mitochondrial genomes, COI, COII, $\mathrm{CytB}$ and NADH mitochondrial genes as well as ITS2 ribosomal region from NCBI database were used in order to construct the phylogenetic trees of Acrididae family. In addition, we experimentally obtained sequences of COI, COII, CytB genes and ITS2 region for more than 40 species in order to compliment the database information. In order to assign the right substitution model to each coding gene, their alighments were parsed by PartitionFinder 2 v2.1.1. All phylogenetic trees were build using Maximum likelihood method realized in IQ-Tree online program $v$ 1.6.10. In addition, we performed the Bayesian analysis using MrBayes program v 3.2.7.

Results: In order to establish phylogenetic relationships between Acrididae subfamilies, we compared complete coding mitochondrial and nuclear ITS2 DNA sequences for more than hundred Acrididae grasshoppers. In addition, we analysed four concatenated mitochondrial genes, COI, COII, CytB and NADH. Preliminary results show that all studied acridids fall into three main phylogenetic groups that include 13 subfamilies: (I) Oxyinae, Hemiacridinae, and Spathosterninae; (II) Melanoplinae; (III) Acridinae, Calliptaminae, Catantopinae, Cyrtacanthacridinae, Eyprepocnemidinae, Euryphyminae, Gomphocerinae, Oedipodinae, and Pezotettiginae. Additional comparison of recent studies to our data reveals a fourth group embracing five more subfamilies: (IV) Marelliinae, Pauliniinae, Leptysminae, Rhytidochrotinae, and Ommatolampidinae. Subfamilies Acridinae, Gomphocerinae and Oedipodinae appears to be polyphyletic and form several independent branches inside one main group that join all three subfamilies together.

Conclusion: Our analysis show that the commonly recognized subfamilies - Acridinae, Gomphocerinae and Oedipodinae appears to be polyphyletic, forming at least 2 new groups of species that may have a subfamily status.

Acknowledgements: The project was supported by the Russian Foundation for Basic Research (No. 17-04-01615). 